\title{
Management Of Post Stroke Seizures
}

\author{
Kavian Ghandehari, MD FLSP RCPC, \\ Director of Comprehensive Stroke Center and Stroke Fellowship Program, Mashhad UM S, Iran. \\ *Corresponding Author: Email: GhandehariK@mums.ac.ir
}

The incidence of seizures in relation to stroke is $8.9 \%$, with a frequency of 10.6 and $8.6 \%$ in haemorrhagic and ischaemic stroke, respectively. In subarachnoid haemorrhage the incidence is $8.5 \%$. Due to the fact that infarcts are significantly more frequent than haemorrhages, seizures are mainly related to occlusive vascular disease of the brain. The general view is to consider stroke-related seizures as harmless complications in the course of a prolonged vascular disease involving the heart and brain. Seizures can be classified as those of early and those of late onset in a paradigm comparable to post-traumatic epilepsy, with an arbitrary dividing point of two weeks after the event. Most early-onset seizures occur during the first day after the stroke. Late-onset seizures occur three times more often than early-onset ones. A first late-onset epileptic event is most likely to take place between six months and two years after the stroke. However, up to $28 \%$ of patients develop their first seizure several years later. Simple partial seizures, with or without secondary generalisation, account for about $50 \%$ of total seizures, while complex partial spells, with or without secondary generalisation, and primary generalised tonic-clonic insults account for approximately $25 \%$ each. Status epilepticus occurs in $12 \%$ of stroke patients, but the recurrence rate after an initial status epilepticus is not higher than after a single seizure. Inhibitory seizures, mimicking transient ischaemic attacks, are observed in $7.1 \%$ of cases. The only clinical predictor of late-onset seizures is the initial presentation of partial anterior circulation syndrome due to a territorial infarct. Patients with total anterior circulation syndrome have less chance of developing epileptic spells, not only due to their shorter life expectancy but also due to the fact that the large infarcts are sharply demarcated in these patients. The optimal timing and type of antiepileptic drug treatment for patients with poststroke seizures is still a controversial issue. Prospective studies in the literature showed that immediate treatment after a first unprovoked seizure does not improve the long-term remission rate. However, because of the physical and psychological influences of recurrent seizures, prophylactic treatment should be considered after a first unprovoked event in an elderly person at high risk of recurrence, taking into consideration the individuality of the patient and a discussion with the patient and his/her family about the risks and benefits of both options latest studies regarding post-stroke seizure treatment showed that 'new-generation' drugs, such as lamotrigine, gabapentin and levetiracetam, in low doses would be reasonable. Although several studies suggest that seizures alter the functional recovery after a stroke, it remains difficult to determine whether or not the occurrence of a second seizure in an untreated stroke patient might hamper the overall outcome. However, repeated seizures and status epilepticus worsen the neurological and mental condition of stroke patienton The decision to initiate antiepileptic drug treatment after a first or a second post-stroke seizure should therefore be individualized, primarily based on the functional impact of the first seizure episode and the patient's preference. Several converging findings suggest that the majority of first-generation antiepileptic drugs, particularly phenytoin, are not the most appropriate choice in stroke patients because of their potential harmful impact on functional recovery and bone health, their suboptimal pharmacokinetic profile and interaction with anticoagulants or salicylates, their greater likelihood to be poorly tolerated, and the lack of level A evidence regarding their specific use in elderly patients. Among the new-generation drugs that do not interact with anticoagulants, antiplatelet agents, or bone health, lamotrigine and gabapentine are the only two drugs that proved to be more effective than immediate-release carbamazepine in elderly patients, providing level A evidence for their use in this indication. In addition, gabapentin remains the only drug that has been specifically evaluated in stroke patients, demonstrating a high rate of long-term seizure freedom. At present, low-dose lamotrigine or gabapentin appears to represent the optimal first-line therapy for post-stroke seizure and epilepsy in elderly patients or in younger patients requiring anticoagulants. However, low-dose extended-release carbamazepine might be a reasonable and less expensive option in patients with appropriate bone health who do not requiring anticoagulat. Based on the stroke management guidelines antiepileptic drugs should not be administered as preventive management in any type of stroke patients without seizure.

Key words: Seizure, Stroke, Management 